\title{
QUANTIFICAR, PADRONIZAR E PLANEJAR: REGISTROS DE CÂNCER E ATENÇÃO ONCOLÓGICA NO BRASIL (1960-1980)
}

Luiz Alves Araújo Neto ${ }^{1}$

Recebido em: 24/04/2021

Aprovado em: 26/05/2021

Resumo: Este artigo discute o processo de criação dos primeiros registros de câncer no Brasil e os debates sobre quantificação, padronização e planejamento da atenção oncológica relacionados à estruturação desses serviços. Argumento que a trajetória conflituosa dos registros de câncer no país exemplifica a tensão entre uma estrutura conceitual da medicina, em mudança durante a segunda metade do século XX, e a realidade institucional da atenção oncológica. A discussão é circunscrita ao intervalo entre 1960 e 1980, período em que os primeiros registros foram criados em São Paulo e Pernambuco, em 1967, seguidos de outros em Porto Alegre, Fortaleza, João Pessoa e Rio de Janeiro. Além disso, esse intervalo foi marcado pelos debates sobre a importância da notificação e registro dos casos de câncer para a construção de uma política de atenção oncológica eficaz, permitindo visualizar as tensões mencionadas no argumento. $\mathrm{O}$ artigo dialoga com os Estudos Sociais da Medicina e a Sociologia da Quantificação, utilizando fontes de caráter técnico, como publicações em periódicos especializados em câncer, relatórios e publicações de instituições médicas e de saúde e documentos institucionais.

Palavras-chave: câncer; registros de câncer; quantificação; padronização; Brasil.

\section{CUANTIFICAR, ESTANDARIZAR Y PLANIFICAR: REGISTROS DE CÁNCER Y ATENCIÓN DEL CÁNCER EN BRASIL (1960-1980)}

Resumen: Este artículo analiza el proceso de creación de los primeros registros de cáncer en Brasil y los debates sobre la cuantificación, estandarización y planificación de la atención oncológica relacionados con la estructuración de estos servicios. Sostengo que la trayectoria conflictiva de los registros de cáncer en el país ejemplifica la tensión entre una estructura conceptual de la medicina, que cambió durante la segunda mitad del siglo XX, y la realidad institucional de la atención del cáncer. La discusión se limita al intervalo entre 1960 y 1980, período en el que se crearon los primeros registros en São Paulo y Pernambuco, en 1967, seguidos de otros en Porto Alegre, Fortaleza, João Pessoa y Río de Janeiro. Además, este intervalo estuvo marcado por debates sobre la importancia de la notificación y registro de los casos de cáncer para la construcción de una política de atención oncológica eficaz, que permita

\footnotetext{
${ }^{1}$ Doutor em História das Ciências e da Saúde. Observatório História e Saúde (Casa de Oswaldo Cruz/Fiocruz). Programa de Pós-Graduação em História das Ciências e da Saúde, bolsista de pós-doutorado (PDR-10/FAPERJ). E-mail: luizalvesan@ hotmail.com. ORCID id: https://orcid.org/0000-0001-7965-2957.
} 
vislumbrar las tensiones mencionadas en el argumento. El artículo dialoga con los Estudios Sociales de la Medicina y la Sociología de la Cuantificación, utilizando fuentes técnicas, como publicaciones en revistas especializadas en cáncer, informes y publicaciones de instituciones médicas y de salud y documentos institucionales.

Palabras clave: cáncer; registros de cáncer; cuantificación; estandardización; Brasil.

\section{QUANTIFY, STANDARDIZE AND PLAN: CANCER REGISTRIES AND CANCER CARE IN BRAZIL (1960-1980)}

Abstract: This article discusses the creation of the first cancer registries in Brazil and the debates on quantification, standardization, and planning of cancer care related to the structuring of these services. This conflicted trajectory of cancer registries, I argue, is an example of the tension between a conceptual structure of medicine, changing during the second half of the 20th century, and the institutional reality of cancer care. The discussion is limited to the interval between 1960 and 1980, when the first registries were created in São Paulo and Pernambuco, in 1967, followed by others in Porto Alegre, Fortaleza, João Pessoa, and Rio de Janeiro. Also, this period was marked by debates about the importance of notification and registration of cancer cases for the construction of an effective cancer care policy. The article is based on Social Studies of Medicine and the Sociology of Quantification, using technical sources, such as publications in journals specialized in cancer, reports, and publications from medical and health institutions and institutional documents.

Keywords: cancer; cancer registries; quantification; standardization; Brazil

\section{Introdução}

Nas últimas décadas, os processos de produção do conhecimento e de tomada de decisões no campo da saúde têm sido cada vez mais pautados pelo uso massivo de dados numéricos, seja para a definição de políticas públicas ou de protocolos clínicos (Adams, 2013; 2016). Esse modelo epistêmico, conectado diretamente à Medicina Baseada em Evidências (EBM) tem como principal prerrogativa o planejamento das ações em saúde a partir das "melhores evidências". Para que seja possível traçar estratégias orientadas por dados numéricos, uma condição necessária é a existência de bases de informação constantes e confiáveis, um problema estrutural ao campo da saúde brasileiro (Araújo Neto, 2020).

O estabelecimento da quantificação como um imperativo à produção de evidências na medicina e na saúde pública remete ao período pós-Segunda Guerra Mundial (Weisz et al., 2007), principalmente devido a uma demanda por regulação da prática médica após episódios drásticos, como o caso da talidomida (Timmermanns \& Berg, 2003). Um resultado relevante desse pensamento quantificado foi a criação dos Registros de Câncer, serviços dedicados à coleta, análise e divulgação de dados estatísticos sobre a doença. Existentes desde o início do 
século XX, esses registros tiveram uma complicada e descontinuada história de institucionalização no Brasil. Atualmente, a literatura nacional e internacional, bem como os documentos de organização da atenção oncológica, considera a atuação desses serviços fundamental para o planejamento de estratégias que visem a reduzir o número de mortes e de novos casos da doença (Micheli et al., 2010; INCA, 2012).

A origem dos registros está diretamente relacionada a mudanças metodológicas e epistemológicas no campo da estatística médica, principalmente ao desenvolvimento dos estudos de morbidade. Diferente da mortalidade, que faz medições a partir do número de óbitos por uma doença, a morbidade é uma variável que mede o processo de adoecimento, abrindo a possibilidade de discussões sobre os fatores socioambientais e biológicos envolvidos na ocorrência de uma doença. Em termos de planejamento, a morbidade serve como dado para estratégias que visam à redução do número de casos, enquanto a mortalidade, da quantidade de mortes.

Em termos de sistematização dos dados, existem dois tipos de registros: os de base populacional $(\mathrm{RCBP})$ e os hospitalares $(\mathrm{RCH})$. O primeiro grupo diz respeito a serviços que coletam dados de uma população em área geográfica delimitada (normalmente um município), e procuram analisar o comportamento da doença no território de levantamento; o segundo, por sua vez, contempla dados de pacientes de hospitais, com análises mais circunscritas e direcionadas à eficiência dos serviços de assistência (diagnóstico, tratamento, reabilitação etc.).

No Brasil, o processo de institucionalização dos registros de câncer foi permeado por debates quanto ao modelo de atenção oncológica estabelecido no país. Desde os anos 1930, e principalmente durante os anos 1950, a estrutura de cuidado aos cancerosos foi constituída tendo como centro hospitais especializados voltados para a realização do diagnóstico precoce e do tratamento dos tumores (Teixeira, 2010). Esse modelo institucional traduzia uma série de mudanças conceituais que aconteceram na medicina do câncer na primeira metade do século XX, e sua permanência na segunda metade teve grande impacto na criação dos registros de câncer no país, e, mais especificamente, na predominância dos registros hospitalares (Araújo Neto, 2019).

Neste artigo, discuto o processo de criação dos primeiros registros de câncer no Brasil e os debates sobre quantificação, padronização e planejamento da atenção oncológica relacionados à estruturação desses serviços. Argumento que a trajetória conflituosa dos registros de câncer, principalmente os de base populacional, no país são exemplares da tensão entre uma estrutura conceitual da medicina, em mudança durante a segunda metade do século XX, e a realidade institucional da atenção oncológica. Circunscrevo a discussão deste artigo 
entre 1960 e 1980, período em que os primeiros registros foram criados em São Paulo e Pernambuco, em 1967, seguidos de outros em Porto Alegre, Fortaleza, João Pessoa e Rio de Janeiro. Além disso, esse intervalo foi marcado pelos debates sobre a importância da notificação e registro dos casos de câncer para a construção de uma política de atenção oncológica eficaz, permitindo visualizar as tensões mencionadas no argumento.

Este artigo explora discussões feitas em minha tese de doutorado, na qual discuti a história conceitual e institucional da prevenção do câncer no Brasil (Araújo Neto, 2019). Aproprio-me de reflexões e debates recorrentes nos Estudos Sociais da Medicina e na Sociologia da Quantificação, como a relação entre o processo de quantificação e os regimes de objetividade na medicina (Cambrosio et al., 2006), as tensões envolvidas em medidas regulatórias da prática médica (Porter, 1995; Timmermanns \& Berg, 2003), e a compreensão da padronização como resultado de um desenvolvimento histórico da episteme médica (Weisz, 2014; Weisz et al., 2007). Utilizo fontes de caráter técnico, como publicações em periódicos especializados em câncer, relatórios e publicações de instituições médicas e de saúde (Organização Mundial da Saúde, Registros de Câncer, Divisão Nacional de Câncer), e documentos institucionais (leis, decretos e regimentos internos).

\section{Estatística médica e os registros de câncer}

A preocupação com o conhecimento estatístico do câncer no Brasil possui uma longa história, remetendo aos primeiros anos do século XX. Em artigo de 1904, no Brazil Medico, Azevedo Sodré destacava a importância de conhecer a frequência (número de casos em uma sequência temporal numa população) da doença no país, a fim de ter dimensão do problema do câncer e da necessidade de organizar uma luta em âmbito nacional (Sodré, 1904). Em edição do Congresso Brasileiro de Higiene de 1928, Eduardo Rabello, chefe da Inspetoria de Câncer, Lepra e Doenças Venéreas, também indicava a subnotificação como um dos problemas para o reconhecimento do impacto social do câncer no Brasil, sendo necessários investimentos em serviços de apuração estatística de mortalidade pela doença (Rabello, 1928).

Somente a partir da Era Vargas, entretanto, começaram a ser criados serviços para a produção de estatísticas sobre os tumores malignos no país. Criado pelo decreto-lei 3.171 de 1941, o Serviço Federal de Bioestatística foi um importante aliado de outras instituições de saúde, como o Serviço Nacional de Câncer (SNC), na produção de estatísticas sanitárias (Fonseca, 2007: 243), embora seu campo de ação fosse limitado, principalmente fora do Distrito Federal e estados com serviços previamente estruturados, como São Paulo e Rio Grande do Sul. 
O próprio SNC, criado em 1942, tinha entre suas atribuições a realização de "estudos, inquéritos e pesquisas sobre epidemiologia, profilaxia, diagnóstico e tratamento do câncer” (Brasil, 1944), sendo responsável por reunir dados das demais instituições dedicadas à doença no país. Nas décadas de 1930 a 1950, os movimentos de organização da luta anticancerosa em diversos estados também tomavam como ponto relevante a produção de estatísticas iniciais sobre a mortalidade por câncer em suas capitais, a fim de legitimar a doença como problema social relevante e presente no Brasil (Araújo Neto, 2018; Araújo Neto \& Teixeira, 2017).

Um aspecto comum aos estudos estatísticos sobre neoplasias realizados no país nesse período dizia respeito à natureza metodológica, indicando dados de mortalidade pela doença nas capitais, por localidade específica ou em seu conjunto (Briggs, 1936; Tramujas, 1948). A partir desses números, alguns trabalhos analisavam a frequência da doença, iniciando a explorar a chamada "patologia geográfica", ou seja, a distribuição dos casos e mortes por localização anatômica, recorte social e região geográfica (Graça, 1944; Alencar, 1951; Merched; Marsillac, 1954). Os dados eram obtidos nos hospitais e enfermarias onde os médicos trabalhavam, sendo, em muitos casos, os únicos serviços disponíveis nas cidades para cuidado aos cancerosos. A partir desses números, os médicos emulavam discursos de defesa do fortalecimento institucional da luta anticancerosa, solicitando mais recursos para o aparelhamento de hospitais e mobilização de campanhas.

Outro ponto importante é o envolvimento profissional com o tema da estatística do câncer. Em teoria, o trabalho de levantamento e análise de dados estatísticos ficava a cargo de médicos sanitaristas, ocupantes de cargo no serviço público (centros de saúde, postos de higiene, ou mesmo no Serviço de Bioestatística). O que se observa na documentação, porém, é a ocupação desses postos principalmente por cirurgiões (caso do Rio de Janeiro, Curitiba, Bahia e Rio Grande do Sul) ou por patologistas (como em Pernambuco e São Paulo). No caso do Serviço Nacional de Câncer, a Seção de Epidemiologia, criada nos anos 1950, teve como primeiros diretores e fundadores os cirurgiões Ugo Pinheiro Guimarães e Jorge de Marsillac.

Marsillac foi um dos principais atores envolvidos com a ampliação dos estudos estatísticos sobre câncer em meados do século XX. Compartilhando da reivindicação de muitas lideranças das associações anticâncer, o médico carioca defendia a criação de serviços específicos para a mensuração do problema do câncer no Brasil, permitindo a definição de sua patologia geográfica. Essas informações, na leitura de Marsillac, seriam fundamentais para planejar os passos da campanha nacional, por exemplo, quais localizações deveriam receber maior atenção das ações educativas (Marsillac, 1965). 
A especialização dos dados de mortalidade por câncer passava diretamente pela consolidação da figura do patologista e dos serviços de anatomia patológica, permitindo a realização de autópsias com maior precisão do diagnóstico diferencial dos tumores e fornecendo laudos mais completos sobre cada paciente, como destacou Achilles Scorzelli Junior, diretor do Departamento Nacional de Saúde, em artigo publicado na Revista Brasileira de Cancerologia, em 1956. O problema, segundo o sanitarista, era que o recurso da anatomia patológica se restringia a poucos centros, além de não haver padronização dos processos de coleta, análise e relatoria das informações, tornando a pesquisa estatística necessariamente falha (Scorzelli Jr., 1956: 74).

A dificuldade na coleta de dados era algo bastante relevante, pois impossibilitava a produção de séries epidemiológicas consistentes. Somando isso à própria dificuldade de diversos médicos em diagnosticar tumor maligno e à falta de um padrão de procedimentos e nomenclaturas para os tumores, o resultado era um conjunto de dados frágeis dentro da própria dinâmica da comunidade médica do período (Araújo Neto, 2019). Esse aspecto é central. Nos estudos discutidos até aqui, a estatística é utilizada para argumentações na dimensão "médicosocial" do câncer, ou seja, afirmar a relevância da doença na agenda sanitária, sendo recorrentemente articulada em discursos públicos de cancerologistas para reivindicar investimentos do poder público (Teixeira, 2010). Entretanto, as estatísticas de mortalidade, nesse período, não compunham um sistema de evidências fixado para a atividade da saúde pública, sendo utilizados para a formulação de uma retórica acerca da relevância do problema do câncer para o país.

Os atores produtores das evidências para a prática clínica eram patologistas, cirurgiões, radiologistas etc., não os estatísticos. Isso não significa que as estatísticas apresentadas em congressos, artigos, palestras e na imprensa eram irrelevantes; mas que os seus usos estavam focados no apoio às mobilizações pela criação de instituições e na organização do combate à doença. A mudança nesse estatuto de evidência está ligada ao desenvolvimento da epidemiologia das doenças crônicas como campo disciplinar e à introdução e consolidação dos estudos de morbidade, ou seja, que buscavam mapear os novos casos de doenças através de dados de diagnósticos, não pelos números de óbitos. A partir desse mapeamento, foi possível a realização de estudos analíticos sobre os fatores de risco e as condições de sociais relacionadas ao processo de adoecimento.

Essa tipologia de pesquisa estatística ganhou bastante força na Europa e nos Estados Unidos principalmente após a Segunda Guerra Mundial, mas possui antecedentes no século XIX. Na segunda metade dos Oitocentos, o Imperial Cancer Research Fund, na Inglaterra, e o 
Ministério da Cultura da Alemanha realizaram levantamentos dessa natureza direcionados às neoplasias em Londres e Hamburgo, respectivamente (Wagner, 1985). Outras iniciativas similares foram realizadas na Suécia, Dinamarca, Espanha e Noruega, mas os estudos de morbidade ganharam maior relevância como evidências para a prática médica a partir da década de 1930, com o desenvolvimento de inquéritos epidemiológicos para doenças crônicas nos Estados Unidos (Weisz, 2014).

Essas pesquisas tinham como base a coleta de dados em diferentes fontes sobre um tema previamente determinado, no caso, doenças crônicas e incapacidades. Esse método de uma "busca ativa" de dados relativos à morbidade serviu de base para uma grande discussão sobre a reforma do cuidado à saúde nos EUA, gerando relatórios sobre prevenção e as formas de atenção aos pacientes (Ibidem). Movido por discussões similares, o Institut National d'Hygiène da França, através de sua Seção de Câncer, organizou a partir de 1943, um Inquérito Permanente de Câncer (Enquête Permanente Cancer - EPC), com o intuito de mapear o papel dos tumores no declínio demográfico do país no período (Ménoret, 2002).

Na direção do EPC estava o cirurgião Pierre Denoix. A partir de 1944, quando os primeiros dados de morbidade foram coletados pelo inquérito, Denoix apontou a importância de padronizar a nomenclatura dos tumores e o procedimento de coleta, a fim de tornar o conjunto de dados mais sistematizado e adequado à produção de análises sobre as populações em questão. A abordagem direcionada a dados de população, como um "estudo de comunidade", também deu ao inquérito prerrogativas para discutir empiricamente temas até então incipientes na medicina, como as relações de hereditariedade em casos de câncer, ou mesmo o impacto real de fatores de risco sobre grupos populacionais.

A repercussão dos estudos de morbidade realizados nos Estados Unidos e na França deu maior legitimidade aos inquéritos permanentes, que, no caso do câncer, passaram a ser realizados por serviços específicos, chamados de registros de câncer. O modelo de registro oriundo dos inquéritos de morbidade foi denominado de base populacional, pois coletava dados de diversos serviços de saúde em regiões delimitadas. Estruturas do mesmo formato foram criadas na Dinamarca, na Finlândia e na Itália (Terracini; Zanetti, 2003). Um dos casos mais interessantes de criação de registros de base populacional ocorreu no Japão, onde, em 19571958, foi implantando um inquérito permanente sobre câncer em Hiroshima e Nagasaki, a fim de avaliar os impactos das bombas atômicas na população sobrevivente ao ataque norteamericano (Okamoto, 2008).

Na América Latina, nas décadas de 1950 e 1960, quinze projetos de criação de registros de câncer foram iniciados, sendo o primeiro deles o de Porto Rico, em 1950, seguido pelos da 
Jamaica (1958), Chile (1959) e México (1960) (Mirra, 2005). No Brasil, as primeiras articulações em torno de inquéritos permanentes partiram de médicos de São Paulo e Pernambuco.

Movidos por essas preocupações, dois médicos brasileiros realizaram estágios em centros de pesquisas em estatísticas do câncer. Em 1956, Antônio Pedro Mirra, cirurgião torácico do Hospital A.C. Camargo, de São Paulo, recebeu uma bolsa de estudos do governo francês para estagiar no Institut Gustave Roussy, onde funcionava o Enquête Permanente Cancer dirigido por Pierre Denoix (Mirra, 2005). No ano seguinte, o patologista pernambucano Adonis de Carvalho, então professor assistente da Faculdade Medicina da Universidade de Pernambuco, viajou aos Estados Unidos, onde realizou estágio no registro implantando no MD Anderson Cancer Center, em Houston, estado do Texas. Os dois médicos tiveram contato com uma mesma modalidade de pesquisa estatística: inquéritos permanentes que coletavam dados sobre a incidência de câncer em hospitais, enfermarias, clínicas, serviços de anatomia patológica, serviços de óbito e laboratórios. As informações eram reunidas em um serviço específico, um Registro de Câncer, responsável pela apuração regular de fichas detalhadas.

Um aspecto que chamou a atenção tanto de Mirra quanto de Carvalho, além da metodologia ampla de coleta, dizia respeito às análises fornecidas pelos boletins dos registros francês e texano, com indicações as quais extrapolavam a patologia geográfica, mapeando possíveis fatores de risco na comunidade, oferecendo prognósticos para procedimentos terapêuticos específicos, sugerindo prioridades para a organização da atenção oncológica na região, entre outros pontos (Mirra, 1959; Carvalho, 1964). Os registros de câncer de base populacional poderiam fornecer dados úteis não somente para alertar a população e o poder público do impacto social da doença, mas também para organizar a "luta anticancerosa", estabelecendo prioridades, estratégias e metas às instituições e atores da atenção oncológica. Ou seja, a partir dos registros seriam organizados sistemas de informação e planejamento capazes de basilar as ações de saúde pública.

Para viabilizar a criação dos registros em São Paulo e Recife, portanto, era necessário estabelecer a importância dos estudos de morbidade entre a comunidade médica brasileira, e afirmar o papel do conhecimento epidemiológico para a organização da luta contra o câncer no Brasil. A trajetória dos dois serviços demonstra diferenças e similaridades regionais na concepção de como a atenção oncológica deveria ser estruturada, como também condições de possibilidades específicas aos próprios atores envolvidos na fundação dos registros de câncer.

\section{A criação dos registros de câncer de São Paulo e Pernambuco}


A criação dos registros de Recife e São Paulo esteve diretamente relacionada a distintas configurações da atenção oncológica em cada cidade, frutos de modelos institucionais e trajetórias pessoais diferenciadas entre paulistas e pernambucanos. De saída, dois aspectos fundamentais merecem destaque: 1) em São Paulo, o registro de câncer foi vinculado ao Departamento de Epidemiologia da Faculdade de Higiene e Saúde Pública da Universidade de São Paulo (USP), enquanto em Recife o serviço foi associado à Cátedra de Anatomia Patológica da Faculdade de Medicina da Universidade de Pernambuco (UFPE); e 2) a fundação do registro paulistano foi circunscrita ao movimento estadual de organização do combate ao câncer, enquanto sua contraparte pernambucana contou com a cooperação do Serviço Nacional de Câncer para o início de suas atividades.

Logo após seu retorno do estágio em Paris, Mirra empreendeu sua primeira tentativa de implantar um registro na capital paulista. Segundo o próprio cirurgião, mesmo que a direção do hospital tivesse reconhecido a importância de um serviço estatístico, a situação financeira da instituição não permitia o financiamento de um registro (Mirra, 1959). Em 1958, dois anos após o retorno de Mirra de sua experiência no Inquérito Permanente de Câncer na França, foi levada uma proposta ao Departamento de Epidemiologia da Faculdade de Higiene e Saúde Pública da USP, sob a justificativa de que "tal iniciativa seria um primeiro passo para o início dos estudos epidemiológicos do câncer em São Paulo", o que se "enquadrava bem nos próprios objetivos daquele departamento de ensino" (RGC-SP, 1964: 7). Novamente, a proposta parece ter sido bem recebida na faculdade, mas outras condições impediriam a concretização do empreendimento naquele momento.

Em participação na II Jornada Brasileira de Cancerologia, realizada em 1961, no Rio de Janeiro, Mirra proferiu uma palestra sobre o que chamou de "diretrizes para estudos epidemiológicos do câncer no Brasil”. Antônio Pedro Mirra salientava o estatuto de problema de saúde pública da doença, chamando atenção para a importância de se conhecer a "situação do câncer no Brasil", para o planejamento das ações de combate:

No entanto, a tarefa de combater o câncer como um problema nacional de saúde pública, exige, de início, o conhecimento da amplitude da doença por meio de levantamentos epidemiológicos, que são básicos. A finalidade imediata desses estudos é permitir que a doença seja caracterizada epidemiologicamente, fornecendo todos os dados necessários para o estabelecimento, principalmente, de medidas profiláticas; e a mediata é estabelecer uma teoria ou filosofia geral sobre a moléstia (Mirra, 1962: 119). 
O discurso de Mirra na Jornada é significativo de um momento de introdução de novas concepções sobre a epidemiologia do câncer, em que as estatísticas sobre a doença agregariam novas funções, como a "determinação dos prováveis fatores etiológicos (fatores carcinogênicos)" e a realização da "profilaxia do câncer, com o afastamento desses fatores causais, assim determinados" (Mirra, 1962: 119). Para o cirurgião paulista, o mecanismo que permitiria esse conhecimento epidemiológico era o inquérito permanente através de um registro geral de câncer, seguindo o modelo implantado por Pierre Denoix na França.

Um aspecto destacado por Mirra dizia respeito à metodologia de coleta dos dados, que envolvia a implantação de um registro de base populacional. Entre os requisitos necessários para a criação de um serviço dessa natureza, segundo ele, constava a existência de uma estrutura estatística prévia que permitisse o conhecimento demográfico da região delimitada para estudo, e de serviços sanitários de naturezas diversas, possibilitando a obtenção da maior variedade e quantidade de dados sobre casos de câncer possível. Ao comentar essa questão metodológica, Mirra estava criticando uma iniciativa do Instituto Nacional do Câncer (INC), que realizava um inquérito epidemiológico desde 1960, coletando dados sobre mortalidade pela doença em hospitais especializados nas capitais dos estados. Na sua concepção:

Os estudos de incidência baseados em clientelas de hospitais são falsos, porque estes constituem o único recurso médico de determinada região. É impossível se obter uma taxa real, devido à impraticabilidade da delimitação de uma população, em que todos os indivíduos fossem exclusivamente clientes desse hospital considerado. É igualmente perigoso tirarmos conclusões sobre a frequência relativa das diferentes localizações de câncer, quando se estuda uma série de doentes de hospital especializado, em determinado período (Mirra, 1962: 120-121).

O debate introduzido por Mirra na comunicação de 1962 é central ao argumento deste artigo. O modelo de registro proposto em São Paulo e Pernambuco tinha como base inquéritos populacionais, através do funcionamento de serviços de saúde integrados. A criação de registros hospitalares seguia outra metodologia de coleta e de análise dos dados, pois era orientada por uma amostra mais circunscrita: clientes e usuários do espaço hospitalar (especializado). A incongruência apontada pelo médico em 1962 acompanhou as discussões sobre os registros e a epidemiologia do câncer nos anos seguintes, principalmente devido à gradativa predominância de registros hospitalares em detrimento daqueles de base populacional.

Na década de 1960, a Organização Mundial da Saúde formou grupos de trabalho para discutir a atenção oncológica em diversos níveis e temas. O primeiro comitê de especialistas reunido pela OMS tinha como objeto de discussão o tema "controle do câncer", com a incumbência de propor diretrizes gerais para a organização da atenção oncológica pelo mundo. 
Entre os onze membros a formar o grupo, constavam Pierre Denoix; Andrew McGarrity, diretor do Registro de Câncer Ginecológico de Sydney, Austrália; e Einar Pedersen, diretor do Registro de Câncer da Noruega, considerado modelar para os serviços de base populacional (McGarrity, 1964; WHO, 1963).

O relatório produzido pelo comitê de especialistas destacava a importância de planejar políticas e programas de atenção oncológica com base na integração entre pesquisa, prevenção, educação e assistência. As atividades de controle deveriam ter como base dados epidemiológicos sobre a doença, principalmente aqueles relacionados à morbidade, a fim de compreender seu comportamento na população e possibilitar pesquisas sobre a carcinogênese e a proposição de métodos preventivos (WHO, 1963).

As duas modalidades de registro, hospitalar e populacional, foram descritas como serviços de objetivos e utilidades distintas, sendo ambos necessários à organização dos programas de controle. No que tange aos hospitalares, o relatório destaca sua importância na avaliação do tratamento e da reabilitação, além de uma estratégia para implantar procedimentos de seguimento (follow-up) dos pacientes. A introdução da noção de que era preciso acompanhar os pacientes de forma sistemática, não somente para a produção de estudos sobre tratamentos, mas também para avaliar os serviços de modo geral, consistiu uma importante mudança nas formas de pensar atenção oncológica. Quanto aos registros de base populacional, o relatório da OMS delegava maior protagonismo na organização dos programas de controle do câncer, devido à sua demanda pela integração institucional e pela compilação de dados que permitiram acompanhar a doença além da escala hospitalar. Entre as funções desse tipo de registro, é destacada sua possível contribuição ao conhecimento epidemiológico e às pesquisas sobre etiologia.

Em 1963, o médico soviético A. V. Chaklin, diretor do registro de câncer na URSS, chefe da Comissão de Câncer da OMS e secretário do comitê de especialistas que elaborara o relatório comentado acima, foi a São Paulo, onde se reuniu com Antônio Pedro Mirra e professores da Faculdade de Higiene e Saúde Pública da USP para discutir o projeto do cirurgião paulista para a criação do registro. Embora não tenha sido localizada documentação escrita sobre a visita de Chaklin, parece seguro supor sua importância para o empreendimento de Mirra. Àquela altura, as professoras Elza Berquó e Maria Lucila Milanesi, ambas da Faculdade de Saúde Pública, e o médico Massaki Udihara, do Hospital A.C. Camargo, também se engajaram no intento de fundar o registro.

No ano anterior, o projeto de inquérito piloto em São Paulo foi levado novamente às direções da Associação Paulista de Combate ao Câncer e da Faculdade de Higiene e Saúde 
Pública da USP. Também foi apresentado a dezessete laboratórios com unidades na capital paulista, e à Fundação de Amparo à Pesquisa do Estado de São Paulo (FAPESP) (RCSP, 1971). A partir do financiamento de todas essas instituições, foi criado o Registro Central de Câncer de São Paulo, sediado na Faculdade de Saúde Pública, iniciando suas atividades em janeiro de 1963. O total de recursos recebidos pelo Registro alcançou o valor de Cr\$ 552.250,00 (quinhentos e cinquenta e dois mil cruzeiros), utilizados na sua instalação e manutenção pelo primeiro ano (RGC-SP, 1964).

De início, a equipe do registro contava com "três médicos, sendo um estaticista, uma secretária, uma assistente social e um grupo de voluntárias" (RGC-SP, 1964: 10). O trabalho consistia na coleta de dados em instituições de saúde, análise do material pelos médicos, e compilação de boletins. O primeiro inquérito foi realizado entre 1963 e 1965, contando com duas fases de coleta: a primeira somente em instituições especializadas, e a segunda contemplando maior diversidade de instituições. Ao total, foram recolhidos números de 32 hospitais (19 gerais, 12 especializados e o A.C. Camargo); um abrigo para idosos; o laboratório de anatomia patológica da Faculdade de Medicina da USP; o serviço de verificação de óbitos do município; e o Departamento de Estatística do Estado de São Paulo (RCSP, 1971: 4-5).

Com os dados do primeiro inquérito, foi possível realizar estudos retrospectivos de morbidade, indicando não somente a quantidade documentada de novos casos (12.961), mas também iniciando o desenho da patologia geográfica do câncer, constatando, por exemplo, a maior incidência de tumores do estômago entre homens, e de mama nas mulheres. A partir desses números, a equipe do registro defendia a manutenção de um registro com patrocínio oficial, ou seja, financiamento público para o funcionamento do serviço, através do governo federal, “em estrita colaboração com as Secretarias de Saúde dos Estados e Municípios (convênios)" (Mirra, 1967: 11).

A atuação da esfera federal, através do Serviço Nacional de Câncer, para a criação de registros pelo país também começou a ser acentuada na década de 1960. A primeira proposta da Seção de Epidemiologia e Estatística, dirigida à época por Ugo Pinheiro Guimarães, consistia em iniciar um registro de tumores na Guanabara (Mello, 1966). O planejamento envolvia um processo similar ao ocorrido em São Paulo, com a coleta de dados hospitalares seguidos pela busca em outros serviços de saúde. A peculiaridade desse projeto em relação aos outros quatro em discussão no país naquele momento (Recife, São Paulo, Porto Alegre e Fortaleza) consistia em ser o único concebido como um dispositivo de um órgão público, no caso, o Instituto Nacional de Câncer (Mello, 1966). 
De modo similar a Mirra, Adonis de Carvalho retornou de seu estágio no M.D. Anderson Hospital, no Texas, e buscou seus pares dedicados à cancerologia para viabilizar na capital pernambucana uma iniciativa igual à que vira nos EUA (Carvalho, 1964). À época, tanto a Clínica de Câncer de Pernambuco, instituição vinculada à Campanha Nacional, quanto a Faculdade de Medicina da Universidade de Pernambuco, onde Carvalho atuava como professor assistente de anatomia patológica, recusaram o projeto de implantação do registro (Mirra, 2005).

Carvalho, como outros personagens envolvidos em empreendimentos para fundação de registros em seus estados, buscou explicitar a importância de tal iniciativa para o combate ao câncer. Sob o prisma da patologia geográfica, o patologista pernambucano coordenou uma mesa redonda na III Jornada Brasileira de Cancerologia, realizada em Recife no ano de 1963. Na ocasião, médicos do Instituto Offir Loiola, de Belém, da Liga Bahiana Contra o Câncer, de Salvador, e da Associação de Combate ao Câncer de Goiás, de Goiânia, também defenderam a manutenção de inquéritos permanentes em serviços, a fim de melhor conhecer a situação da doença em seus estados (AO, 1963).

Em 1965, quando da realização do I Encontro Nacional dos Coordenadores da Campanha Nacional Contra o Câncer, Adonis de Carvalho apresentou um projeto para criação do Registro de Câncer de Pernambuco. O texto lido pelo patologista detalhava diversos aspectos necessários para o início das atividades do registro, desde a metodologia de coleta e análise dos dados até os materiais mínimos para seu funcionamento cotidiano (armários, fichas etc.).

Em consonância com o posicionamento de Mirra, Carvalho imaginava que a contribuição de um registro repousava em possibilitar discussões sobre a prevenção da doença a partir do mapeamento dos diferentes fatores de risco para tipos distintos de câncer. Para isso, defendia maiores investimentos para estudos de morbidade através de inquéritos de base populacional, apontando que o adoecimento em longo prazo relacionado às doenças crônicas tornava a inferência da morbidade pela mortalidade um movimento analítico falho (Carvalho, 1965: 58).

No que se referia às fontes de informações para o registro, Adonis de Carvalho indicava quatro tipos: a Clínica de Câncer de Pernambuco; os serviços de patologia das instituições de previdência social; serviços privados de patologistas; e as cadeiras de anatomia patológica e cancerologia da Faculdade de Medicina de Pernambuco (Carvalho, 1965: 59). Essa organização seria privilegiada em relação a outros cenários locais, segundo Carvalho, devido à amplitude de dados coletados pelo Serviço de Anatomia Patológica da escola médica pernambucana. A cátedra ocupada por Barros Coelho, tendo Bertoldo Kruse de Arruda como professor adjunto e 
Adonis como assistente, teria a capacidade de compilar informações sobre todo o estado de Pernambuco.

No que diz respeito à equipe necessária para o funcionamento do registro, o projeto de Carvalho também apresentava mais detalhamentos do que as propostas de Mirra para São Paulo. Seriam necessários dois médicos com experiência em saúde pública e/ou cancerologia; quatro auxiliares de estatística; dois datilógrafos; e um servente. O financiamento, a princípio, viria do Serviço Nacional de Câncer e da Faculdade de Medicina de Pernambuco, instituição que seria responsável pelo registro.

Em 1967, o Registro de Câncer de Pernambuco começou suas atividades sob a coordenação de Bertoldo Kruse de Arruda, contando com recursos do Serviço Nacional de Câncer e o apoio das principais instituições de saúde pernambucanas: a Universidade Federal de Pernambuco, a Secretaria de Saúde de Pernambuco e a Sociedade Pernambucana de Combate ao Câncer. Seu primeiro inquérito foi realizado entre 1967 e 1968, contando com informações de trinta e oito hospitais (Hospital de Câncer, cinco maternidades, um sanatório para tuberculosos, uma ala odontológica, cinco manicômios, vinte e cinco hospitais gerais), treze laboratórios privados de patologia, e catorze laboratórios privados de hematologia (CRP, 1970: 5). A partir dos dados coletados, foram elaboradas análises retrospectivas sobre mortalidade (1952-1968) e morbidade (1967-1968) por câncer em Recife.

Os primeiros resultados do inquérito realizado pelo registro pernambucano permitiram iniciar a discussão que marcaria a epidemiologia do câncer naquele estado: a relação entre a grande incidência de câncer do colo do útero e os problemas de desenvolvimento socioeconômico na região. Entre os casos coletados entre mulheres, $25 \%$ dos informados em 1967 (199 novas ocorrências) e 50\% dos notificados em 1968 (304) eram da região cervical do útero (RCP, 1970: 9-10). Nos anos seguintes, Kruse de Arruda e Carvalho intensificariam a discussão sobre os aspectos socioeconômicos do câncer do colo do útero e a necessidade de ações de prevenção e diagnóstico precoce para reduzir a incidência da doença.

A partir de 1969, os registros de São Paulo e Recife iniciaram trabalhos com maior continuidade, estabelecendo uma produção relevante de dados sobre a morbidade e a mortalidade por câncer em suas regiões. A trajetória de criação desses serviços, acompanhadas pelos pares médicos do período, serviu de modelo para diversas iniciativas em âmbitos regionais e nacional, incluindo a tentativa de estabelecer um plano nacional para a fundação de registros de câncer nos anos 1970, comandado pela Divisão Nacional de Câncer (nova nomenclatura atribuída ao Serviço Nacional de Câncer a partir do governo Médici). 
A multiplicação de projetos e inquéritos iniciais para a implantação de registros de câncer em diversas capitais pelo país (Porto Alegre, Fortaleza, Salvador, João Pessoa, Rio de Janeiro, Niterói) trouxe à tona as incongruências entre a proposta de inquéritos populacionais e a realidade da atenção oncológica no Brasil no período. Entre os pontos que se destacavam, estava a dificuldade em obter cooperação das instituições de assistência e a falta de padronização na nomenclatura e nos procedimentos referentes ao diagnóstico do câncer no país. Para o primeiro ponto, os médicos recorriam fortemente à prerrogativa de notificação compulsória estabelecido pelo Código Nacional de Saúde, de 1961, mas também reivindicavam maior participação do poder público para legitimar a obrigatoriedade de informar os novos casos de neoplasias. Quanto ao segundo ponto problemático, a articulação dos médicos envolvia o convencimento dos próprios pares, através de artigos, livros e palestras sobre a importância da padronização para a credibilidade das informações e a garantia de estudos mais acurados sobre a epidemiologia do câncer.

\section{A ampliação dos registros de base populacional e o planejamento da atenção oncológica}

Em janeiro de 1961, um decreto do presidente Juscelino Kubistchek regulamentou o Código Nacional de Saúde, diferenciando a atuação da saúde pública e da previdência social (Brasil, 1961). Entre as prerrogativas do Ministério da Saúde, constava a notificação compulsória de diversas doenças, inclusive o câncer. Os médicos envolvidos com a criação dos registros de câncer tinham grande interesse na efetivação da legislação de notificações, pois um dos principais problemas apresentados nos primeiros inquéritos dizia respeito ao que era chamado de "falta de cooperação" das instituições de assistência e dos próprios clínicos. Ao discutir o tema em artigo publicado na revista Arquivos de Oncologia, Antônio Pedro Mirra demonstrou descontentamento com a situação, afirmando que a regulação não era suficiente para aumentar as notificações. Seria necessário convencer o médico de sua importância:

É ilusório esperar que todos os médicos cooperem nesse inquérito; haverá sempre os refratários. Para que esse número de refratários seja pequeno, o único meio é o de exigir o mínimo de obrigações aos médicos. A experiência tem demonstrado que toda disposição legislativa autoritária, no sentido de cooperação do médico, não tem dado melhores resultados do que a colaboração espontânea. É necessário que o médico se convença da importância desses estudos e de sua colaboração (Mirra, 1963: 69).

A questão colocada por Mirra e outros atores é central na história de criação dos registros de câncer. Por um lado, estava em jogo o processo de regulação da prática clínica pelo 
Estado orientado por um sistema de objetividade estatística, que demandaria a produção de dados para análise e planejamento das ações em saúde. Esse processo, embora em curso desde o final do século XIX no Brasil (Camargo, 2015), era marcado pelo elevado índice de subnotificação por parte das instituições de assistência. De modo geral, a ampliação da importância das estatísticas na produção do conhecimento em saúde, bem como a crescente noção de que era necessário embasar ações médicas em evidências epidemiológicas, aumentou significativamente a pressão para movimentos regulatórios, tanto por parte dos Estados quanto pelas comunidades médicas nacionais (Weisz et al., 2007; Timmermans; Berg, 2003).

Por outro lado, repousava sobre esse tema a própria viabilidade de manutenção dos registros de câncer, afinal, os serviços dependem da alimentação de dados pelas instituições de saúde. Observando esse ponto, a organização da saúde pública brasileira não favorecia ao funcionamento dos registros de base populacional, iniciados em São Paulo e Recife, e propostos no Rio de Janeiro, Porto Alegre, Fortaleza, Goiânia, Salvador e Belém. O fato de a assistência ao câncer ser essencialmente filantrópico-privada, bem como de os registros não serem serviços do Ministério da Saúde, tornavam a integração de estatísticas sanitárias um procedimento bastante complicado. Nem os registros possuíam autoridade para exigir o fornecimento de dados para as instituições (a notificação compulsória era prerrogativa do Ministério), nem a malha hospitalar existente no país se comunicava necessariamente com os serviços de saúde pública.

Pensando nas possibilidades de fortalecer as estatísticas de câncer e viabilizar a criação dos registros, a Seção de Epidemiologia e Estatística do Serviço Nacional de Câncer elaborou um planejamento de trabalho com o objetivo de "promover o levantamento epidemiológico do câncer em todo o País" (Conceição, 1967: 15). Para tanto, indicava o plano, era necessária a criação de um registro no estado da Guanabara, vinculado ao SNC, e de um Registro Geral de Câncer, responsável por coletar e organizar os dados fornecidos pelos demais serviços existentes no Brasil. Também deveriam ser implantados registros regionais, que dependeriam da articulação entre o órgão federal e as associações estaduais de combate ao câncer (Conceição, 1967: 16). Um passo inicial para essa aproximação ocorreria através de palestras, reuniões e distribuição de publicações às instituições interessadas na luta anticancerosa.

A organização pensada pela Seção de Epidemiologia e Estatística do SNC era diferente da existente em Recife e São Paulo, onde os registros estavam vinculados a escolas médicas, não aos institutos. Entretanto, como diversos estados seguiram uma trajetória de centralização das atividades de combate nas instituições de câncer, era plausível propor a implantação de registros dessa forma. Seguindo esse modelo, foram criados cinco serviços de estatística das 
neoplasias: Rio de Janeiro (1970), Fortaleza (1971), Paraíba (1971), Alagoas (1972) e Rio Grande do Sul (1972) (Mirra, 2005). Nos dois primeiros, a proposta dos registros era restrita aos dados de morbidade e mortalidade das capitais dos estados. Em João Pessoa e Maceió, a proposta era coletar números nas capitais e realizar avaliações sobre o comportamento da doença nos estados, sob a premissa de que a maior parte dos doentes buscava atendimento nessas cidades. O Rio Grande do Sul caracterizava um caso diferenciado, pois a criação de um registro partira de municípios do interior que já possuíam sociedades de cancerologia estabelecidas, como Pelotas e Santa Maria.

É importante destacar que todos esses serviços se propunham a funcionar como registros de base populacional, mesma metodologia projetada em Pernambuco e São Paulo. Convencer os médicos e instituições de saúde de que a notificação dos novos casos não era somente uma obrigação legal, mas também parte importante da produção do conhecimento sobre a doença, consistia, portanto, uma atividade constante dos coordenadores de registros, e mesmo do órgão federal, que mudou de nome em 1969: de Serviço Nacional de Câncer, passou a se chamar Divisão Nacional de Câncer (DNC).

Em 1970, a DNC, através da Seção de Epidemiologia e Estatística, realizou um encontro com coordenadores de registros e associações de combate ao câncer, o I Encontro Nacional sobre Registros de Câncer, no Recife. O evento visava ao compartilhamento das experiências de implantação dos serviços e elaborar um planejamento para difundir os registros pelo país. O evento foi organizado por Maria Berila Conceição, da DNC, Bertoldo Kruse de Arruda (PE), Antônio Pedro Mirra (SP), Monteiro Leite (do Instituto Offir Loiola, no Pará), Haroldo Juaçaba (Instituto do Câncer do Ceará), Antônio Carneiro Arnaud (Fundação Napoleão Laureano, da Paraíba), Anibal Silvany Filho (Hospital Aristides Maltez, Bahia), e José Nestor Paiva (Clínica de Câncer do Sergipe). Contou também com representantes de importantes instituições de saúde: Divisão Nacional de Epidemiologia e Estatística da Saúde; Organização Pan-Americana da Saúde (OPAS), Centro Regional de Estatística de Saúde do Nordeste (CRESNE), Instituto Nacional de Previdência Social (INPS), e Fundação Instituto Oswaldo Cruz (Fiocruz).

O tom principal das discussões realizadas no evento se direcionou à padronização do modelo de registro de câncer a ser adotado, dos procedimentos de coleta, análise e divulgação das estatísticas, e das nomenclaturas utilizadas nas fichas dos pacientes. As conclusões e recomendações do evento, minutas e relatórios das sessões, além de um texto normativo sobre a criação de registros, foram compiladas em um documento da Divisão Nacional de Câncer, Registros de Câncer-organização, publicado em 1972. 
A introdução do documento final produzido pelos participantes do evento é significativa do pensamento médico-sanitário sobre o câncer no período. A atuação dos registros foi enquadrada no documento congressista como de fonte de informação para todos os setores envolvidos nas ações de controle do câncer, sendo um dos elementos para a composição da "luta anticancerosa":

O controle do câncer requer a adoção de medidas de planejamento, assistência, educação e pesquisa, motivando, em todas as partes do mundo, inclusive no Brasil, o desenvolvimento de campanhas de combate ao câncer. É uma luta na qual se incorporam todos os elementos de uma sociedade. Os cientistas pesquisadores se empenham em descobrir a causa do mal. Os clínicos procuram encontrar as melhores armas de diagnóstico e terapia. Os epidemiologistas investigam as causas e fatores que de algum modo possam influir sobre a ocorrência da doença nos diversos grupos populacionais. Os sanitaristas orientam o público sobre os exames e cuidados que devem ser adotados para prevenir o mal. As autoridades, por dispositivos legais, determinam a observância de medidas que visam à prevenção. Enfim, os leigos contribuem para a luta anticancerosa aprendendo e divulgando os ensinamentos das campanhas educativas, prestando serviços de qualquer natureza e, principalmente, contribuindo com recursos financeiros (...). Todos estes participantes integram um exército em luta contra o mesmo inimigo - o Câncer. A todos os combatentes uma arma é indispensável para sua ação eficaz - a INFORMAÇÃO (DNC, 1972: 7).

Essa função social atribuída aos serviços de estatística era o ponto de partida para uma argumentação que ressaltasse sua importância às ações dos serviços de saúde do período. Ela também demonstra como a implantação desses registros estava imbricada à construção conceitual do controle do câncer no Brasil. Os problemas em sua institucionalização também refletiam a continuidade da visão de combate estabelecida na Campanha Nacional dos anos 1950/1960. Ao pontuar a proposta de uma rede de registros regionais de câncer vinculados à DNC, o documento listou as funções específicas desses serviços, a partir de campos de atuação: estatística, planejamento, pesquisa, educação e assistência (DNC, 1972: 10-11).

O funcionamento dos registros, fosse de forma isolada ou em rede, dependia absolutamente da notificação dos casos e da coleta e organização dos dados fornecidos pelos serviços de saúde. A primeira etapa consistia na obtenção das fichas pelos médicos ou instituições, elaboradas visando à padronização de nomenclaturas e da estrutura de informações sobre cada paciente. Caberia aos registros enviar a hospitais, enfermarias, clínicas, laboratórios, ambulatórios e outros centros de saúde as fichas. O segundo momento era o preenchimento desse material, considerada a parte mais problemática de todo o trabalho: eram demandadas informações sobre o indivíduo (nome, idade, sexo, "cor", estado civil, nacionalidade e naturalidade, profissão, endereço, dados do serviço que encaminhara a ficha) e o diagnóstico 
(localização anatômica, data, e base do diagnóstico - anatomia patológica, citologia, radiologia, hematologia etc.). O principal problema nesse aspecto era o preenchimento, que não seguia um padrão claro, dificultando a compilação precisa dos dados.

O documento do Encontro sobre Registros de Câncer sugeria, à maneira como fizeram os serviços de São Paulo e Pernambuco, o uso da Classificação Internacional de Doenças (CID), um dos sistemas classificatórios, junto ao TNM, adotados pela OMS para a produção de conhecimento estatístico em saúde. Após a coleta das fichas, caberia ao registro arquivá-las, seguindo critérios que fossem mais adequados ao serviço e à capacitação dos funcionários. A orientação do documento era utilizar o mesmo material (fichas compradas com a IBM) e atender aos padrões de nomenclatura e preenchimento. Através dos dados coletados nas fichas, seria apurada a incidência geral, distribuição de casos por localização anatômica, incidência por grupos (idade, sexo, etnia, profissão etc.), distribuição dos casos por extensão do tumor, distribuição histopatológica (tipo de tecido afetado) (DNC, 1972: 26).

O ponto de chegada desse processo era a publicação de análises estatísticas sobre morbidade e mortalidade por câncer em sua região de ação. Para isso, eram requeridas "boa qualidade das informações"; "uniformidade"; e "comparabilidade dos dados" (DNC, 1972: 27), a fim de conferir credibilidade ao material produzido pelos registros. No planejamento da Divisão Nacional de Câncer, a implantação de um Registro Geral permitiria a compilação de todos os dados regionais em intervalos de seis meses e a realização de análises sobre a situação da doença no Brasil. Também durante o evento, foi elaborado o modelo de termo de convênio a ser celebrado entre o Ministério da Saúde e as instituições interessadas em implantar registros de câncer, prevendo subvenção financeira do governo federal.

Por um lado, toda a mobilização em torno da criação e manutenção dos registros de câncer exprimia claramente uma preocupação com o papel da estatística (e do estatístico) na ordem de produção do conhecimento médico. A ideia de que não era possível planejar a atenção oncológica sem conhecer a situação epidemiológica da doença no país ganhou especificidade em relação às décadas anteriores através da transformação dos estudos de morbidade em evidência para a prática médica. De certa forma, a defesa dos registros demandava repensar a prática cotidiana dos cancerologistas e dos demais profissionais ligados à "luta anticancerosa". Por outro lado, o processo de implantação dos registros representa a constituição histórica, gradual e complexa, de uma forma de enquadrar o câncer através dos conhecimentos da epidemiologia, ampliando o papel da prevenção e propondo medidas que extrapolavam o espaço clínico-hospitalar. 
No intervalo de cinco anos, sete registros de câncer estavam postos em funcionamento no Brasil (Recife, São Paulo, Rio de Janeiro, Fortaleza, Paraíba, Alagoas e Rio Grande do Sul). O caminho para estabelecimento de registros de base populacional parecia bem pavimentado, principalmente com os resultados do encontro de Recife, em 1970. Entretanto, o cenário mais amplo das políticas de câncer no período indicava outro caminho: na década de 1970, a ênfase repousava na realização de campanhas preventivas para cânceres ginecológicos através de parcerias com entidades filantrópicas e secretarias estaduais, e no fortalecimento das instituições assistenciais, como parte da política de expansão da cobertura previdenciária do regime militar (Teixeira; Porto; Habib, 2012: 378). Nesse sentido, o Programa Nacional de Controle do Câncer (PNCC), criado em 1973, incentivava a criação de registros hospitalares, sem indicar planejamento para registros de base populacional.

Os anos posteriores ao PNCC foram bastante complicados para a manutenção dos registros de base populacional. Com escassez de recursos, os serviços do Rio de Janeiro, da Paraíba, de Alagoas e do Rio Grande do Sul foram desativados, deixando pouca documentação referente ao seu funcionamento e dados. Entre os remanescentes, o registro de Fortaleza teve vida um pouco mais curta, durando uma década, com a formulação de alguns estudos sobre a incidência do câncer na capital cearense (Oliveira, 2017). Em São Paulo e Pernambuco, a parceria com as faculdades de medicina ofereceu maior solidez aos registros, que começaram a divulgar estudos com estatísticas de morbidade e mortalidade por câncer. Também no caso paulista e pernambucano, a entrada de uma nova instituição no cenário de pesquisas sobre as neoplasias foi relevante: o Instituto Ludwig de Pesquisas sobre Câncer (ILPC), de Zurique, na Suíça, estabeleceu auxiliou os registros em suas atividades.

Embora houvesse profundas dificuldades na manutenção dos registros de câncer de base populacional, a partir da segunda metade da década de 1970 estudos epidemiológicos importantes foram desenvolvidos pelos serviços, incluindo a participação em pesquisa intercontinental realizada pela OMS, Cancer Incidence in Five Continents (IARC, 1976). Gradativamente, a pesquisa epidemiológica sobre a doença ganhava maior espaço nos círculos da cancerologia, e publicações descrevendo a "situação do câncer no Brasil”" foram elaboradas, ao mesmo passo em que os registros hospitalares eram multiplicados pelo país e era implantando um Registro Nacional de Patologia Tumoral (Mirra, 2005). 


\section{Considerações finais}

A trajetória dos registros de câncer no Brasil entre 1960 e 1980 permitem visualizar dois processos que alternaram entre o paralelismo e a concorrência. No âmbito conceitual, os projetos de criação dos serviços estatísticos representavam a materialização institucional do processo de quantificação da medicina e de transformação dos dados numéricos em evidência para a prática clínica e o planejamento em saúde, aspectos atualmente consolidados na ciência médica e na saúde pública. Em termos institucionais, a discussão sobre a implantação de registros de base populacional e hospitalares demarca como um modelo de atenção oncológica estabelecida entre os anos 1930 e 1950 teve continuidades importantes nas políticas de câncer e práticas de cuidado.

Para o debate contemporâneo sobre notificação de doenças e produção de dados, tema central na atual pandemia de Covid-19, considero que este estudo contribui para a compreensão de uma incongruência. A concorrência entre os dois processos, conceitual e institucional, criou um cenário no qual uma parcela importante do campo médico e da saúde consideram a quantificação, a padronização e o planejamento imperativos centrais à produção do conhecimento e à tomada de decisões, mas, na prática cotidiana, a produção de dados numéricos confiáveis e estáveis é bastante difícil. Em resumo, é possível dizer que se acredita na importância dos números, mas não é possível produzi-los de maneira adequada.

No caso do câncer, essa trajetória foi marcada por outros processos importantes nas décadas seguintes ao estudado aqui. A criação do Registro Nacional de Patologia Tumoral, nos anos 1980; de grupos de pesquisa sobre epidemiologia do câncer em universidades e institutos; de registros de base populacional vinculados a secretarias estaduais de saúde nos anos 1990; e de um sistema nacional de registros hospitalares nos anos 2000 representaram esforços importantes para romper com a incongruência entre estrutura conceitual e realidade institucional na atenção oncológica brasileira. Outro exemplo é a publicação de estatísticas anuais sobre a doença pelo Instituto Nacional de Câncer, iniciada em 1992 e mantida com regularidade. Apesar disso, o problema da subnotificação e da qualidade das bases de dados ainda são pautas centrais às estatísticas da doença no país.

Finalmente, a trajetória dos registros de câncer no Brasil possibilita explorar discussões relevantes aos Estudos Sociais da Medicina e à Sociologia da Quantificação. Um exemplo substancial é o processo de construção social dos padrões, ou seja, a observação de que a transformação de determinados métodos, nomenclaturas e práticas em um "padrão ouro" não é um resultado evidente do desenvolvimento científico, mas resultado de negociações que 
envolvem exatamente a relação entre estrutura conceitual e realidade institucional discutida ao longo deste artigo. Aprofundar esse debate no âmbito da história da medicina e da saúde pode ser uma chave útil para compreendermos os percursos intricados da prática médica e do planejamento em saúde no Brasil ao longo do tempo e no presente.

\section{Referências bibliográficas}

ADAMS, Vincanne. "Evidence-Based Public Health: subjects, profits, erasures". In: BIEHL, João \& Petryna, Adriana. When People Come First: Critical Studies in Global Health. Princeton and Oxford: Princeton University Press, 2013. pp. 54-90.

ADAMS, Vincanne. Metrics: What Counts in Global Health. London: Duke University Press, 2016.

A.O. Relatório sobre a Segunda Jornada Brasileira de Cancerologia. Arquivos de Oncologia. Volume V, n.1, 1963. 279-282.

ARAÚJO NETO, Luiz Alves. A notificação de doenças no Brasil: um problema histórico.

Boletim da Sociedade Brasileira de História da Ciência, 24, 2020.

ARAÚJO NETO, Luiz Alves. Prevenção do câncer no Brasil: mudança conceitual e continuidade institucional no século XX. Tese de Doutorado - PPGHCS Fiocruz. Rio de Janeiro, 2019. 362f.

ARAÚJO NETO, Luiz Alves e TEIXEIRA, Luiz Antonio. De doença da civilização a problema de saúde pública: câncer, sociedade e medicina brasileira no século XX. Boletim do Museu Paraense Emílio Goeldi. Ciências Humanas, v. 12, n.1, p. 173-188, jan-abr, 2017.

ARAÚJO NETO, Luiz Alves. O problema do câncer no Ceará: cancerologia, controle do câncer e a atividade coletiva da medicina. Rio de Janeiro: Luminária Acadêmica, 2018.

BRASIL. Decreto n ${ }^{0}$ 49.974-a, de 21 de janeiro de 1961. Regulamenta, sob a denominação de Código Nacional de Saúde, a Lei n ${ }^{\circ}$.312, de 3 de setembro de 1954, de normas gerais sobre defesa e proteção da saúde.

BRASIL. Decreto no 15.971, de 4 de julho de 1944. Aprova o Regimento do Serviço Nacional de Câncer, do Departamento Nacional de Saúde. Disponível em https://www.planalto.gov.br/legislacao. Acesso em: 07/05/2015.

BRIGGS, Luís. Estatística: à margem da mortalidade pelo câncer no Rio de Janeiro. Arquivos de Higiene. Publicação do DNSP. Ano 1, n. 2, setembro de 1927. p. 321-405.

CAMARGO, Alexandre de Paiva Rio. Dimensões da nação: uma análise do discurso estatístico da Diretoria Geral de Estatística (1872-1930). Revista Brasileira de Ciências Sociais, v. 30, n. 87, 2015. p. 79-95.

CAMBROSIO, Alberto et al. Regulatory objectivity and the generation and management of evidence in medicine. Social Science \& Medicine, 63 (2006): 189-199.

CANCER REGISTRY OF PERNAMBUCO (CRP). Cancer Registry of Pernambuco (19671968). Rio de Janeiro: Serviço Nacional de Câncer, 1970.

CARVALHO, Adonis de. Ante-projeto do serviço de Registro de Câncer de Pernambuco. Revista Brasileira de Cancerologia, 1965, 22(12): 57-63.

CARVALHO, Adonis de. Câncer como problema de saúde pública. Boletim Informativo da Clínica de Câncer do Recife, 1964 (3): p. 6-7.

CONCEIÇÃO, Maria Berilla. Plano de trabalho da seção de epidemiologia e estatística do Serviço Nacional de Câncer. Revista Brasileira de Cancerologia. Vol. 23, n. 34. Setembro, 1967. p. 12-19. 
DIVISÃO NACIONAL DE CÂNCER (DNC). Registros de Câncer - organização. Série Mensagens aos Médicos. Rio de Janeiro: Divisão Nacional de Câncer, 1972.

FONSECA, Cristina. Saúde no Governo Vargas: dualidade institucional de um bem público. Rio de Janeiro: Editora Fiocruz, 2007.

GRAÇA, Döllinger da. O problema do câncer no Brasil: observação pessoal no câncer da mama e do útero. Brazil Medico, 32/33: 298-304. 1944.

IARC. Cancer Incidence in Five Continents. V. III. Lyon: International Agency for Research on Cancer, 1976.

INCA (Instituto Nacional do Câncer). ABC do Câncer: abordagens básicas para o controle do câncer. Rio de Janeiro: MS/INCA, 2012. 2ª edição.

MARSILLAC, Jorge de. Patologia Geográfica. Revista Brasileira de Cancerologia, 21(29), 1965: 15-28.

MCGARRITY, Andrew. Cancer control. International Journal of Gynecology \& Obstetrics. Volume 2, issue 2-3: 88-100, 1964.

MELLO, Eder Jansen de. Aperfeiçoamento das estatísticas de morbidade do câncer no estado da Guanabara mediante a criação de um serviço de registro de tumores. Revista Brasileira de Cancerologia. Vol. 22, n. 32. Dezembro, 1966. p. 5-14.

MÉNORET, Marie. The Genesis of the Notion of Stages in Oncology: The French Permanent Cancer Survey (1943-1952). Social History of Medicine, v. 15, n. 2, 2002. p. 291-302.

MERCHED, Naim; MARSILLAC, Jorge de. Câncer e estatística. Revista Brasileira de Cancerologia, 11(12), 1954. p. 65-80.

MICHELI, Andrea. "Evaluating the outcomes of cancer control". In: ELWOOD, Mark J.; SUTCLIFFE, Simon B. (org.). Cancer control. New York: Oxford University Press, 2010. p. 341-362.

MIRRA, Antônio Pedro. Câncer e estatística. Revista Paulista de Medicina. 54 (2), 1959. p. 107-115.

MIRRA, Antonio Pedro. Diretrizes para os estudos epidemiológicos do câncer no Brasil. Arquivos de Oncologia. Vol. V, n. 1. 1965. p. 65-72.

MIRRA, Antônio Pedro. Diretrizes para os estudos epidemiológicos do câncer no Brasil.

Boletim de Oncologia (Revista Brasileira de Cirurgia), vol. 43, n. 2, 1962. p. 119-124.

MIRRA, Antonio Pedro. Registro de Câncer: importância e problemática. Revista Brasileira de Cancerologia. Vol. 23, n. 34. Setembro, 1967. p. 8-11.

MIRRA, Antônio Pedro. Registros de Câncer no Brasil e sua História. São Paulo: Ministério da Saúde, 2005.

OKAMOTO, Naoyuki. A history of the cancer registration system in Japan. International Journal of Clinical Oncology. 13, 2008: 90-96.

OLIVEIRA, Thayane Lopes de. "Você finge não ver e isso dá câncer": controle do câncer de mama no Ceará, 1960-1980. Dissertação de Mestrado. Programa de Pós-Graduação em História das Ciências e da Saúde. Casa de Oswaldo Cruz/Fiocruz. Rio de Janeiro, 2017.

PORTER, Theodore. Trust in Numbers: the pursuit of objectivity in Science and Public Life. Princeton: Princeton University Press, 1995.

RABELLO, Eduardo. O problema do câncer. Conferência feita no $2^{\circ}$ Congresso Brasileiro de Hygiene, em Bello Horizonte. Annaes do Segundo Congresso Brasileiro de Hygiene. Rio de Janeiro: Pimenta de Mello \& Cia, 1928.

REGISTRO DE CÂNCER DE SÃO PAULO (RCSP). Registro de Câncer de São Paulo Inquérito Piloto (1963-1965). São Paulo: Faculdade de Saúde Pública da Universidade de São Paulo, 1971.

REGISTRO DE CÂNCER DE SÃO PAULO. Incidência do câncer no município de São Paulo em 1969. Brasília: Divisão Nacional de Câncer, 1975. 
REGISTRO GERAL DE CÂNCER SÃO PAULO (RGC-SP). Boletim Informativo do Serviço de Registro Geral de Câncer São Paulo. Ano 1, n. 1, 1964.

SCORZELLI JR., Achilles. Câncer como causa de morte. Revista Brasileira de Cancerologia, 13(15), 1956: 71-89.

TEIXEIRA, Luiz Antonio da Silva; PORTO, Marco e HABIB, Paula Arantes. Políticas públicas de controle de câncer no Brasil: elementos de uma trajetória. Cad. Saúde Colet., 2012, Rio de Janeiro, 20 (3): 375-80.

TEIXEIRA, Luiz Antonio. O controle do câncer no Brasil na primeira metade do século XX. História, Ciências, Saúde - Manguinhos. Rio de Janeiro: Vol. 17, Supl. 1, 2010.

TERRACINI, Benedetto; ZANETTI, Roberto. A short history of pathology registries, with emphasis on cancer registries. Präventivmed. Series: Epidemiology. 48 (3), 2003: p. 3-10.

TIMMERMANS, Stefan; BERG, Marc. The Gold Standard: the challenge of evidence-based medicine and standardization in health care. Philadelphia: Temple University Press, 2003.

TRAMUJAS, Armando. Aspectos estatísticos da mortalidade por câncer em Curitiba. Revista Brasileira de Cancerologia, 1948, 2(3): 69-77.

WAGNER, G. Cancer registration: historical aspects. IARC Scientific Publication, 66, 1985: 312.

WEISZ, George et al. The Emergence of Clinical Practice Guidelines. The Milbank Quarterly, v. 85, n. 4, 2007, p. 691-727.

WEISZ, George. Chronic Disease in the Twentieth Century: a history. Baltimore: John Hopkins University Press, 2014.

WHO. Cancer Control. First report of an expert committee. Genebra: WHO, 1963. 